\title{
ОЦЕНКА ЭФФЕКТИВНОСТИ ТЕРАПИИ ДЕНОСУМАБОМ И ДИНАМИКА ФУНКЦИОНАЛЬНОГО СТАТУСА У ПАЦИЕНТОК СТАРЧЕСКОГО ВОЗРАСТА С ТЯЖЕЛЫМ ОСТЕОПОРОЗОМ
}

\author{
Мачехина Л.В.', Дудинская Е.Н.', Браилова Н.В.', Онучина Ю.С'., Шарашкина Н.В., ${ }^{1}$ Ткачева О.Н.'
}

'ФГАОУ ВО «Российский национальный исследовательский медицинский университет имени Н.И. Пирогова» Минздрава России, ОСП Российский геронтологический научно-клинический центр, Москва

Деносумаб, полностью человеческое моноклональное антитело к лиганду RANK, эффективно снижает резорбцию кости, ингибируя связывание RANK-L с RANK. Исследования эффективности и безопасности деносумаба у лиц старше 75 лет малочисленны и не учитывают функциональный статус пожилого пациента.

ЦЕЛЬ: оценить эффективность антиостеопоротической терапии деносумабом и динамику функционального статуса у женщин старческого возраста (75 лет и старше) в течение 12 месяцев.

МЕТОДЫ: обследовано 64 женщины (средний возраст 82+4,6 лет) с тяжелым сенильным остеопорозом и наличием множественных переломов тел позвонков. Всем пациенткам проводили определение уровня сывороточного кальция, 25 ОН витамина Д, измерение МПК (с оценкой Т-критерия) проксимального отдела бедренной кости и поясничного отдела позвоночника с помощью двухэнергетической рентгеновской абсорбциометрии (DXA, GE Lunar), оценку активности в повседневной жизни (индекс Бартел), оценку скорости ходьбы, тест «встань и иди» до начала антиостеопоротической терапии и через 12 месяцев терапии деносумабом. Все пациентки получали терапию препаратами кальция и витамина ДЗ.

Средняя продолжительность терапии деносумабом составила 13,7士1,4 месяца.

PEзУЛЬTATЫ: через 12 месяцев терапии деносумабом отмечено увеличение Т-критерия в области поясничного отдела позвоночника $(-3,1 \pm 0,5$ на исходном уровне и -2,0 $\pm 0,8$ через 12 месяцев, $\mathrm{P}=0,05$, тест Уилкоксона) и в области проксимального отдела бедренной кости $(-3,4 \pm 0,8$ в начале исследования

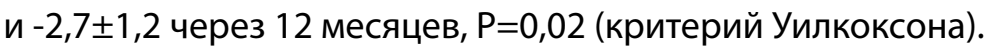

У всех пациенток в течение 6 месяцев от начала исследования достигнут целевой уровень витамина

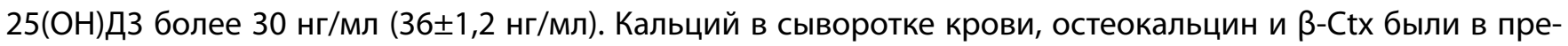
делах нормы. Вновь возникших патологических переломов в течение 12 месяцев терапии деносумабом не наблюдалось.

Все пациентки с тяжелым остеопорозом до начала терапии были зависимы от посторонней помощи в различной степени: до терапии выраженная зависимость наблюдалась у 12 женщин, умеренная зависимость - у 30 пациенток и легкая зависимость - у 22 женщин. Через 12 месяцев терапии повседневная активность у большинства пациенток возросла: выраженная зависимость наблюдалась лишь у 9 женщин, умеренная зависимость - у 26 пациенток и легкая зависимость - у 29 женщин.

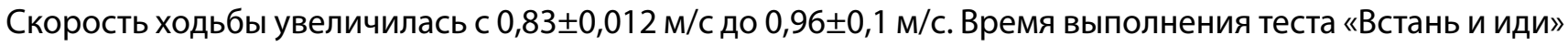
уменьшилось с 14,2 $\pm 0,16$ сек до $11,5 \pm 1,01$ сек.

ВЫвОды: результаты нашей работы подтверждают немногочисленные литературные данные, свидетельствующие о значительной эффективности деносумаба при лечении сенильного остеопороза у лиц старше 75 лет в отношении плотности кости и отсутствии новых низкоэнергетических переломов. Кроме этого, терапия деносумабом в течение 12 месяцев у гериатрических пациентов с умеренным и выраженным снижением функционального статуса приводит к его улучшению, а также увеличению активности пациента старческого возраста. 